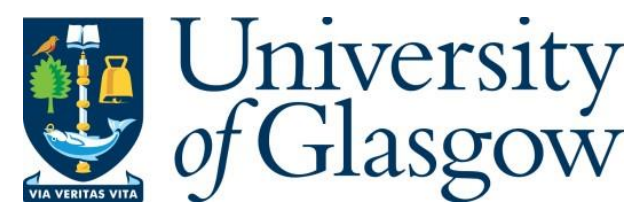

Casado, E., La Civita, M., Vilaplana, M. and McGookin, E. W. (2017) Quantification of Aircraft Trajectory Prediction Uncertainty Using Polynomial Chaos Expansions. In: 2017 IEEE/AIA 36th Digital Avionics Systems Conference, St. Petersburg, FL, USA, 17-21 Sep 2017, ISBN 9781538603659.

There may be differences between this version and the published version. You are advised to consult the publisher's version if you wish to cite from it.

http://eprints.gla.ac.uk/154682/

Deposited on: 4 January 2018

Enlighten - Research publications by members of the University of Glasgow http://eprints.gla.ac.uk 


\section{Quantification of Aircraft Trajectory Prediction Uncertainty using Polynomial Chaos Expansions}

\author{
Enrique Casado \\ Airspace \& Operational \\ Efficiency - Europe \\ Boeing Research \& Technology - \\ Europe \\ Madrid, Spain \\ enrique.casado@boeing.com
}

\author{
Marco La Civita \\ Airspace \& Operational \\ Efficiency - Europe \\ Boeing Research \& Technology - \\ Europe \\ Madrid, Spain \\ marco.lacivita@boeing.com
}

\author{
Miguel Vilaplana \\ Airspace \& Operational \\ Efficiency - Europe \\ Boeing Research \& Technology - \\ Europe \\ Madrid, Spain \\ miguel.vilaplana@boeing.com
}

\author{
Euan W. McGookin \\ Senior Lecturer \\ Division of Aerospace Sciences \\ School of Engineering \\ University of Glasgow \\ Glasgow G12 8LT, Scotland, UK \\ euan.mcgookin@glasgow.ac.uk
}

\begin{abstract}
A novel approach to quantify the uncertainty associated with any aircraft trajectory prediction based on the application of the Polynomial Chaos (PC) theory is presented. The proposed method relies on univariate polynomial descriptions of the uncertainty sources affecting the trajectory prediction process. Those descriptions are used to build the multivariate polynomial expansions that represent the variability of the aircraft state variables along the predicted trajectory. A case study compares the results obtained by a classical Monte Carlo approach with those generated by applying the so-called arbitrary Polynomial Chaos Expansions (aPCE). The results provided herein lead to conclude that this new methodology can be used to accurately quantify trajectory prediction uncertainty with a very low computational effort, enabling the capability of computing the uncertainty of the individual trajectories of a traffic sample of thousands flights within very short time intervals.
\end{abstract}

Keywords- aircraft trajectory prediction; uncertainty quantification; polynomial chaos; generalized PCE; arbitrary PCE.

\section{INTRODUCTION (HEADING 1)}

The concept of Air Traffic Management (ATM) gathers all activities related to provide the means and services to ensure safe and ordered air traffic operations [1]. The ATM capabilities include the Air Traffic Control (ATC) functionality which represents all functions aiming at maintaining the separation among aircraft, and among aircraft and ground obstacles, to avoid the risk of collisions at any time and circumstances. This safety objective must also enable an efficient air traffic flow while meeting as much as possible the user preferences. The Air Traffic Flow Management (ATFM) functionalities represent the processes and procedures required to regulate the flow of aircraft minimizing potential airspace congestions without jeopardizing safety. Aircraft trajectory prediction is the enabler that provides the core means to perform all ATM functionalities.

A trajectory can be defined as the time evolution of the aircraft state, represented by the position of the aircraft's center of mass with respect to a known system of reference (e.g., Geodetic Reference System [GRS]), and other relevant state

This project has received funding from the SESAR Joint Undertaking under grant agreement No 699274 under European Union's Horizon 2020 research and innovation programme. variables such as airspeeds or aircraft mass. A trajectory prediction is considered as a forecast of the aircraft trajectory represented by a chronologically ordered sequence of aircraft states. To compute a prediction, it is required to define a mathematical model of the aircraft motion. In ATM, it is common to adopt the point-mass model approach, which reduces the problem to a 3 degrees-of-freedom (DOF) problem [2]. This model-based approach required from additional information to return a trajectory prediction: (a) weather information that includes wind forecast and atmosphere conditions (temperature and pressure); (b) aircraft performance data that provide information regarding the drag, thrust and fuel consumption for the considered aircraft at the specified flight conditions; and (c) aircraft intent information that represents the command and control inputs issued by the pilot or the Flight Management System (FMS) to steer the aircraft according to the filed Flight Plan (FP).

The assumptions behind of such kind of model-based trajectory prediction approaches, and the stochastic nature of the phenomena involved in the prediction process, bring deviations between the actual and predicted trajectories. The formers, denoted as epistemic uncertainty [3], represent the imperfections or lack of knowledge regarding the considered aircraft motion model. The latters, denoted as variability uncertainty, represent the propagation of the inputs randomness into the outputs. Thus, although high fidelity models can be used constraining the epistemic uncertainty, the intrinsic stochastic behavior of the model inputs leads to unavoidable prediction uncertainties, represented by the time evolution of the aircraft state variable randomness.

Nowadays, the ATM is shifting worldwide from the current surveillance-based airspace-driven paradigm to the new trajectory-based network-centric one [4][5][6], in which advanced Decision Support Tools (DST) will help humans along the Collaborative Decision Making (CDM) process. In this new environment, it is expected that advanced ATM applications will exploit trajectory prediction uncertainty to provide improved and more reliable indications that help to increase the capacity and efficiency of the system. 
This paper proposes an innovative mathematical method to quantify trajectory prediction uncertainty based on a multivariate polynomial description of prediction outputs that relies on univariate polynomial descriptions of the considered sources of uncertainty. This formulation will provide a formal representation of the prediction uncertainties that can be used afterwards by ATM automation tools.

The remaining of this paper is organized as follows. Section II provides insights into the trajectory prediction process, introducing the concept of prediction accuracy and giving details about some techniques used in the literature to quantify prediction uncertainty. Section III exposes the mathematical formulation of the PC theory with special attention to the data driven process defined by the aPCE approach. Section IV describes the application of the PC theory to the problem of quantifying the aircraft trajectory prediction uncertainty. Section $\mathrm{V}$ proposes a case study to assess the suitability of the proposed methodology compared to a classical Monte Carlo approach. Finally, Section VI outlines the main remarks of this research and future steps.

\section{AIRCRAFt TRAJECTORY PREDICTION}

\section{A. Introduction}

The cornerstone of all ATM functionalities is the capability of accurately predict aircraft trajectories so that DST can provide reliable indications about how to manage the traffic flow efficiently while preserving safety. This capability will become especially relevant in the future Trajectory Based Operations (TBO) environment, in which trajectory information will be shared among a variety of automation systems in a timely, robust, and reliable manner.

Despite the fact that not all Trajectory Predictors (TP) are designed and implemented similarly and do not make use of the same data sources, for ATM interoperability purposes, the FAA/EUROCONTROL Action Plan 16 proposed a generic TP model, including a set of common architectural concepts upon which TBO capabilities could be developed [7]. The core of the models is the Trajectory Prediction Process (TPP), which encompasses the process of obtaining a computed trajectory (CT) by means of a set of methods and algorithms implemented by the Trajectory Computation Engine (TCE). The input to the TPP is the Flight Script (FS), which can be defined as a blueprint of the planned trajectory compliant with the applicable ATC restrictions and, as much as possible, with the user preferences. The TE requires information about weather and aircraft performance to properly instantiate the mathematical representation of the Aircraft Motion Model (AMM).

\section{B. Trajectory Prediction Accuracy}

Prediction accuracy can be defined as a measurement of the discrepancy between the actual and predicted aircraft states along the considered time interval. For a coherent accuracy assessment, it is required to define the data sampling at which the actual and predicted trajectories are to be compared, and the metrics to be applied to measure such accuracy [8]. The data sampling basically ensures that the variables to be compared represent the aircraft state at a given trajectory event (e.g., end of the climb phase at the Top of Climb [TOC]) or at any other specified instant. It essentially represents the temporal accuracy and indicates the time difference between the predicted and actual instants at which the trajectory event is achieved (e.g., time at which the aircraft reached the TOC). The metrics to be applied represent spatial accuracy and capture the vertical and horizontal distances between the predicted and actual aircraft positions at a fix time.

Typical metrics are the cross- and along-track deviations that can be measured with respect to the closest flight segment or a time-matched segment [9], or the estimated time of arrival (ETA) deviation at a given waypoint [10].

\section{Quantification of Trajectory Prediction Uncertainty}

Trajectory prediction uncertainty can be described as the estimated amount, or percentage, by which a predicted trajectory may potentially differ from the actual trajectory. Dissimilar to trajectory accuracy, trajectory uncertainty cannot be obtained by comparing predictions with actual trajectories because it represents an a priori estimation of such probable deviations based on the knowledge and quantification of the sources of uncertainty impacting the prediction. The process of identifying, characterizing, quantifying, tracing and managing the uncertainty in simulated and real systems is known as Uncertainty Quantification (UQ). UQ deals with the assessment of system input/output response behavior (forward uncertainty propagation) and with the estimation of unknown system parameters (i.e., parameter calibration) by computing the bias correction comparing simulated outputs with real experiments (inverse uncertainty quantification).

In trajectory prediction, the common approach is the forward uncertainty propagation, in which the effect of prediction inputs variability is assessed by computing a set of predictions and a series of statistics based on them. Covariance analyses provide the standard deviation of the distribution that characterizes the variation between the nominal and stochastic predictions as a function of the statistical properties of the inputs. The covariance of the parametric and time-varying disturbances, and the autocorrelation of time-varying ones, can be used to build the covariance matrix of any state variable throughout the trajectory prediction [11].

\section{POLYNOMIAL CHAOS THEORY}

\section{A. Introduction}

A Polynomial Chaos Expansion (PCE) is a mechanism to represent a stochastic random variable $z$ by means of a basis of polynomials of another random variable $\xi[12]$.

$$
z \sim f(\xi)
$$

Equation (1) is read as " $z$ is distributed as $f(\xi)$ ", meaning that the probabilistic distribution that represents the stochastic behavior of $z$ is the same as that representing $f(\xi)$. Given distributions for $z$ and $\xi$, there is no a unique function $f$ that satisfies (1). Unlike, there is a variety of functions that could build the random variable $z$ form the selected germ $\xi$. Furthermore, additional representations are plausible using different germs rather than $\xi$. 
PCE is an approach that expands function $f$ in a polynomial series. The polynomial basis $\left(\psi_{i}\right)$ is a set of orthogonal polynomials with respect to the probability density (PDF) function $\Gamma(\xi)$ of the germ $\xi$,

$$
\left\langle\psi_{i}, \psi_{j}\right\rangle=\int \psi_{i}(\xi) \cdot \psi_{j}(\xi) \cdot \Gamma(\xi) d \xi=\delta_{i j}
$$

where $\delta_{i j}$ is the Kronecker delta.

A very important feature of such polynomial basis is that all polynomials of order $i \geq 1$ have zero mean due to their orthogonality with $\psi_{0}$, and that the covariance between two polynomials of different order is zero (uncorrelated polynomials) because of $\left\langle\psi_{i}, \psi_{j}\right\rangle=0 ; \forall i \neq j$. Thus, it is possible to build an orthonormal basis of polynomials by assuming $\left\langle\psi_{i}, \psi_{i}\right\rangle=1 \forall i$.

Making use of the orthonormal basis of polynomials, the relationship stated in (1) can be formulated as follows:

$$
z=\sum_{i=1}^{\infty} a_{i} \psi_{i}(\xi)
$$

Polynomial Chaos (PC) theory based on Wiener-Askey theory of homogeneous chaos [13], defines the $i$-th mode as the combination of $a_{i}$ (mode strength) and $\psi_{i}(\xi)$ (mode function).

Since there are many possible functions $f$ that may satisfy (1), there a multiple PCEs for a given $z$ using a determined germ $\xi$ that will only differ in the mode strengths.

The exposed formulation is also valid for representing stochastic random variables in which $\xi$ is a vector comprising $N$ multiple germs $\left\{\xi_{1}, \xi_{2}, \ldots, \xi_{N}\right\}$. In this case, the stochastic variable $z$ will be represented by the following expansion:

$$
z=\sum_{i=1}^{\infty} b_{i} \phi_{i}\left(\xi_{1}, \xi_{2}, \ldots, \xi_{N}\right)
$$

Where $\phi_{i}$ is a tensor product of the univariate polynomial bases regarding each $\xi_{j}$.

$$
\begin{gathered}
\phi_{i}\left(\xi_{1}, \xi_{2}, \ldots, \xi_{N}\right)=\prod_{j=1}^{N} \psi_{j}^{\alpha_{j}^{i}}\left(\xi_{j}\right) \\
\sum_{j=1}^{N} \alpha_{j}^{i} \leq p, \quad i=1, \ldots, N
\end{gathered}
$$

The multivariate index $\alpha_{j}^{i}$ represents the combinatory of all possible products of $\psi_{j}^{k}$ (polynomial of order $k$ belonging to the polynomial basis of germ $\xi_{j}$ ), where $p$ is the number of expansion terms, and depends on the number of germs $N$ and the order of the expansion $d$ (order at which the expansion is truncated) as follows:

$$
p=\frac{(N+d) !}{N ! d !}
$$

The index $\alpha_{j}^{i}$ is a $p \times N$ matrix that represents the corresponding expansion degree for germ $j$ in expansion term $i$. For the sake of clarity, following Table I illustrates the matrix $\alpha_{j}^{i}$ for the case of $N=2, d=2$ and $p=6$.

The unknown coefficients $a_{i}$ (univariate) or $b_{i}$ (multivariate) of the PCE can be obtained by projecting each variable on the polynomial basis (Galerkin projection method [14]), or by estimating them form a limited number of simulations applying regression techniques (probabilistic collocation method [15]).

TABLE I. MATRIX OF INDEXES $\alpha_{j}^{i}$

\begin{tabular}{|c|c|c|c|c|}
\hline \multicolumn{2}{|c|}{$\alpha_{j}^{i}$} & $i$ & $\sum_{j=1}^{n} \alpha_{j}^{i}$ & $\phi_{i}=\prod_{j=1}^{N} \psi_{j}^{\boldsymbol{\alpha}_{j}^{i}}\left(\xi_{i}\right)$ \\
\hline 0 & 0 & 1 & 0 & $\boldsymbol{\psi}_{\mathbf{1}}^{\mathbf{0}} \cdot \boldsymbol{\psi}_{2}^{\mathbf{0}}$ \\
\hline 0 & 1 & 2 & 1 & $\boldsymbol{\psi}_{\mathbf{1}}^{\mathbf{0}} \cdot \boldsymbol{\psi}_{2}^{\mathbf{1}}$ \\
\hline 1 & 0 & 3 & 1 & $\boldsymbol{\psi}_{\mathbf{1}}^{\mathbf{1}} \cdot \boldsymbol{\psi}_{2}^{\mathbf{0}}$ \\
\hline 1 & 1 & 4 & 2 & $\boldsymbol{\psi}_{\mathbf{1}}^{\mathbf{1}} \cdot \boldsymbol{\psi}_{2}^{\mathbf{1}}$ \\
\hline 2 & 0 & 5 & 2 & $\boldsymbol{\psi}_{\mathbf{1}}^{\mathbf{2}} \cdot \boldsymbol{\psi}_{2}^{\mathbf{0}}$ \\
\hline 0 & 2 & 6 & 2 & $\boldsymbol{\psi}_{\mathbf{1}}^{\mathbf{0}} \cdot \boldsymbol{\psi}_{2}^{\mathbf{2}}$ \\
\hline
\end{tabular}

The statistical moments that characterize the behavior of the random variable $z$ can be analytically obtained from the computed coefficients $a_{i}$ (univariate) and $b_{i}$ (multivariate) of the corresponding PCEs as shown in following Table II.

TABLE II. MEAN AND VARIANCE OF THE RANDOM VARIABLE $Z$

\begin{tabular}{|c|c|c|}
\hline & Univariate PCE & Multivariate PCE \\
\hline Mean & $\mu_{z}=a_{1}$ & $\mu_{z}=b_{1}$ \\
\hline Variance & $\sigma_{z}^{2}=\sum_{i=2}^{p} a_{i}^{2}$ & $\sigma_{z}^{2}=\sum_{i=2}^{N} b_{i}^{2}<\phi_{i}^{2}>$ \\
\hline
\end{tabular}

\section{B. Generalized PCE}

Originally, PC theory was applied only to Gaussian stochastic processes, although rapidly its applicability was extended to more generic stochastic problems. The generalized PC (gPC) approach [16] considers that both inputs and outputs can be represented by known random distributions. For those other non-normal distributions, basis of polynomials that allow the identification of the corresponding PCE have already been identified. Table III shows the relationship between germs' distributions and their associated orthogonal polynomials of the Askey scheme.

This approach considers that the germs $\left\{\xi_{1}, \xi_{2}, \ldots, \xi_{N}\right\}$ are independent, identically distributed and can be represented by one of the distributions presented Table III. 
TABLE III. GERMS' DISTRIBUTIONS AND RELATED POLYNOMIAL BASIS

\begin{tabular}{|c|c|}
\hline Germ $\boldsymbol{\xi}$ & Polynomial Basis \\
\hline Gaussian & Hermite \\
\hline Gamma & Laguerre \\
\hline Beta & Jacobi \\
\hline Uniform & Legendre \\
\hline Poisson & Charlier \\
\hline Binomial & Krawtchouk \\
\hline Negative binomial & Meixner \\
\hline Hypergeometric & Hahn \\
\hline
\end{tabular}

\section{Arbitrary PCE}

The gPC method requires the exact knowledge of the germs, which is not the case in most real systems. To manage incomplete or/and implicit distributions only defined by their statistical moments, the use of Gram-Schmidt orthogonalisation led to the definition of the arbitrary PC (aPC) method [17].

In aPC, the exact probability description of the germs is not strictly necessary. For a finite-order expansion, only a finite number of statistical moments is required. This method enables data-driven applications of the PC theory, in which data samples with limited size allow the inference of the polynomial description of the system outputs as a result of the impact of uncertain inputs described by arbitrary distributions (e.g., discrete, continuous, or discretized continuous).

This approach requires from the construction of the polynomial basis representing the stochastic behavior of each germ $\xi_{i}$ by means of the statistical moments calculated from data. To obtain such basis, orthogonality needs to be imposed. In addition, the coefficient of the term of highest order is set to 1 for of all polynomials of the basis. Considering that, the expansion of the polynomial of order $k$ belonging to the polynomial basis of germ $\xi_{i}$ can be expressed as

$$
\begin{gathered}
\psi_{i}^{k}=\sum_{m=0}^{k} c_{m}^{(k)} \xi_{i}^{m} \\
c_{k}^{(k)}=1
\end{gathered}
$$

The zero-order polynomial can be immediately obtained:

$$
\psi_{i}^{0}=c_{0}^{(0)} \xi_{i}^{0}=1
$$

The basis is successively constructed for all remaining polynomials of the $\xi_{i}$ basis up to the selected order $d$ by solving the following system of equations:

$$
\begin{gathered}
\int c_{0}^{(0)} \cdot\left[\sum_{m=0}^{k} c_{m}^{(k)} \xi_{i}^{m}\right] \cdot \Gamma\left(\xi_{i}\right) d \xi_{i}=0 \\
\int\left[\sum_{m=0}^{1} c_{m}^{(1)} \xi_{i}^{m}\right] \cdot\left[\sum_{m=0}^{k} c_{m}^{(k)} \xi_{i}^{m}\right] \cdot \Gamma\left(\xi_{i}\right) d \xi_{i}=0
\end{gathered}
$$

$$
\begin{gathered}
\int\left[\sum_{m=0}^{k-1} c_{m}^{(k-1)} \xi_{i}^{m}\right] \cdot\left[\sum_{m=0}^{k} c_{m}^{(k)} \xi_{i}^{m}\right] \cdot \Gamma\left(\xi_{i}\right) d \xi_{i}=0 \\
c_{k}^{(k)}=1
\end{gathered}
$$

This system of equation can be rearranged if the first equation is introduced into the second, the first and the second into the third and so on. Considering the condition defined by (9), the system of equations turns into:

$$
\begin{gathered}
\int \sum_{m=0}^{k} c_{m}^{(k)} \xi_{i}^{m} \cdot \Gamma\left(\xi_{i}\right) d \xi_{i}=0 \\
\int \sum_{m=0}^{k} c_{m}^{(k)} \xi_{i}^{m+1} \cdot \Gamma\left(\xi_{i}\right) d \xi_{i}=0 \\
\vdots \\
\int \sum_{m=0}^{k} c_{m}^{(k)} \xi_{i}^{m+k-1} \cdot \Gamma\left(\xi_{i}\right) d \xi_{i}=0 \\
c_{k}^{(k)}=1
\end{gathered}
$$

The $m$-th statistical moment of the germ $\xi_{i}$ can be defined as:

$$
\mu_{k}=\int \xi_{i}^{m} \cdot \Gamma\left(\xi_{i}\right) d \xi_{i}
$$

Thus, the system of equations posed in (12) turns into:

$$
\begin{gathered}
\sum_{m=0}^{k} c_{m}^{(k)} \mu_{m}=0 \\
\sum_{m=0}^{k} c_{m}^{(k)} \mu_{m+1}=0 \\
\vdots \\
\sum_{m=0}^{k} c_{m}^{(k)} \mu_{m+k-1}=0 \\
c_{k}^{(k)}=1
\end{gathered}
$$

or alternatively written in a matrix form as:

$$
\left[\begin{array}{cccc}
\mu_{0} & \mu_{1} & \cdots & \mu_{k} \\
\mu_{1} & \mu_{2} & \cdots & \mu_{k+1} \\
\vdots & \vdots & \vdots & \vdots \\
\mu_{k-1} & \mu_{k} & \cdots & \mu_{2 k-1} \\
0 & 0 & \cdots & 1
\end{array}\right]\left[\begin{array}{c}
c_{0}^{(k)} \\
c_{1}^{(k)} \\
\vdots \\
c_{k-1}^{(k)} \\
c_{k}^{(k)}
\end{array}\right]=\left[\begin{array}{c}
0 \\
0 \\
\vdots \\
0 \\
1
\end{array}\right]
$$

The coefficients $c_{m}^{(k)}$ of each of the polynomials belonging to the basis of the germ $\xi_{i}$ can be computed if and only if all moments of the $\xi_{i}$ distribution up to order $2 k-1$ are finite. This ensures that the matrix in (15) is not singular and, therefore, the linear system is solvable. Hence, only the capability of computing the $2 k-1$ moments of the $\xi_{i}$ distribution is required to 
apply aPC, avoiding the need of having an explicit description of the probability density function associated to the $\xi_{i}$ distribution [18].

\section{TRAJECTORY PREDICTION UQ USING PCE}

\section{A. Kinematic Aircraft Motion Model}

The problem of predicting an aircraft trajectory requires the formal definition of an AMM. Assuming a point-mass model, the mathematical representation of the AMM, formed by the navigation, forces, kinematics and mass equations, can be expressed using the space-state formulation as:

$$
\dot{\boldsymbol{X}}=f(\boldsymbol{X}, \boldsymbol{u}, \boldsymbol{E}, \boldsymbol{\Delta}, t)
$$

Where $\boldsymbol{X}$ is the state vector composed by the true airspeed $\left(\mathrm{v}_{\mathrm{TAS}}\right)$, aerodynamic yaw angle $\left(\gamma_{\mathrm{TAS}}\right)$, aerodynamic path angle $\left(\chi_{\text {TAS }}\right)$, aircraft mass $(\mathrm{m})$, longitude $(\lambda)$, latitude $(\varphi)$ and geodetic altitude (h); $\boldsymbol{u}$ is the control vector formed by the throttle coefficient $\left(\delta_{\mathrm{T}}\right)$, the aerodynamic bank angle $\left(\mu_{\mathrm{TAS}}\right)$ and the lift $(\mathrm{L}) ; \boldsymbol{E}$ is the environmental model vector including the local temperature $(\theta)$ and pressure $(\delta)$ ratios, gravity model $(\mathrm{g})$ and wind components $(\mathbf{w}) ; \boldsymbol{\Delta}$ is the configuration vector defined by the landing gear $\left(\delta_{\mathrm{LG}}\right)$, high lift devices $\left(\delta_{\mathrm{HL}}\right)$ and speed breaks ( $\left.\delta_{\mathrm{SB}}\right)$ settings; $t$ is the time; and $\dot{\boldsymbol{X}}$ is the derivative of $\boldsymbol{X}$ respect to the time $t$. Given the initial conditions, the control parameters $\boldsymbol{u}$, the environmental model $\boldsymbol{E}$ and the configuration parameters $\boldsymbol{\Delta}$, it is possible to integrate the AMM to obtain a trajectory $\boldsymbol{X}$.

The inverse formulation of this problem leads to a constrained mechanical model. This approach transforms the Ordinary Differential Equations (ODE) system stated in (16) into a Differential Algebraic Equations (DAE) system by the definitions of three motion constraints $\left(g_{i}\right)$.

$$
\begin{gathered}
g_{i}(\boldsymbol{X}, \boldsymbol{u}, \boldsymbol{E}, \boldsymbol{\Delta}, t)=0 \\
i=\{1,2,3\}
\end{gathered}
$$

The motion constraints are the generalization of any possible mathematical input that the AMM could accept. However, not all possible sets of three motion constraints determine a wellposed system of equations. The Aircraft Intent Description Language (AIDL) [19] establishes the rules that ensures the formulation of a solvable system with a unique solution. In addition, the AIDL requires the definition of three configuration constraints that determine the aerodynamic configuration of the aircraft at each flight condition (i.e., the position of the landing gear, high lift devices and speed breaks).

The set of constraints that enable the computation of a trajectory prediction formally describe the Aircraft Intent (AI) instance associated to such trajectory. Whereas, the AI is defined as an unambiguous description of how the aircraft will be guided during the time interval for which a predicted trajectory is computed, defining a unique trajectory.

A complete trajectory can thus be described by a chronologically ordered sequence of Operations (OP) that represent the AI segments between two consecutive triggers (i.e., trajectory events). Each OP represents a solvable DAE system, while the complete trajectory can be formulated as a sequential succession of OPs (i.e., DAE systems). The process of solving such a sequence of DAE systems is known as Sequential DAEs Resolution (SDR) [20].

\section{B. Sources of prediction uncertainty}

According to the mathematical formation of the AMM exposed in (16), the following architecture depicted in Fig. 1 shows the different datasets required to compute a predicted trajectory.

The TCE implements the algorithms required to integrate the AMM, while the AI sets the motion and configuration constraints required to properly pose a solvable DAE system. The Initial Conditions (IC) are the values of the aircraft state variables at the first instant of the trajectory from which the prediction will be computed. The Trajectory Prediction Infrastructure (TCI) also includes an aircraft performance model (APM) and an Earth and weather model (EWM). The former provides information about the drag, thrust and fuel consumption of the selected aircraft, while the latter describes the Earth surface, gravity, atmosphere conditions and wind field.

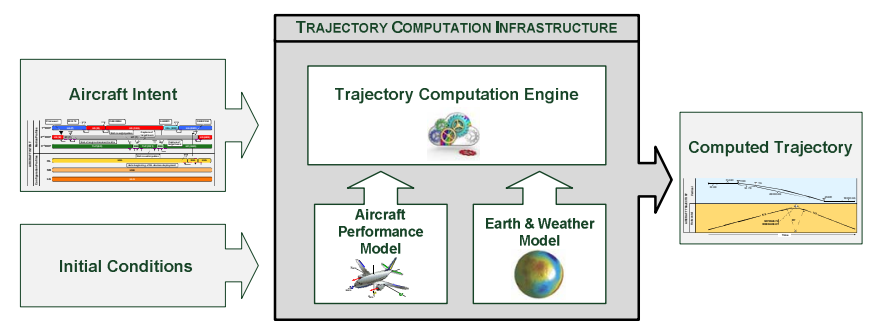

Fig. 1. Trajectory Prediction Infrastructure.

The main advantage of using the proposed approach is the capability to decouple the sources of uncertainty, enabling separate and uncorrelated analyses of their individual influences. According to this formulation, the uncertainty sources affecting the process of predicting an aircraft trajectory can be classified as:

- IC uncertainties, which consider the deviations between the actual and assumed initial values of the aircraft state variables.

- AMM uncertainties, which represent the differences between the real aircraft behavior and the mathematical system of equations that models it.

- APM uncertainties, which collect all inaccuracies of the models used to represent the actual aircraft performance.

- EWM uncertainties, which include the errors introduced by the considered earth model with respect to the actual Earth surface and gravity, and also, the intrinsic stochasticity associated to any weather forecast.

- AI uncertainties, which identify the variations on how the aircraft is finally operated compared to the original plan. The AI can be described by a chronologically ordered sequence of operations. Each operation 
represents a set of command and control actions that determine a unique aircraft behavior during a certain time interval defined between to trajectory events (e.g., between a transition altitude and the cruise altitude). Differences between planned and executed Operations and/or between planned and actual executions intervals shape the AI uncertainties. These uncertainties also account for differences between the pilot/FMS behavioral models used for trajectory prediction and the actual guidance strategy applied by that pilot/FMS.

Table IV explicitly summarizes all possible sources of uncertainty.

TABLE IV. IDENTIFICATION OF SOURCES OF UNCERTAINTY

\begin{tabular}{|c|c|}
\hline $\begin{array}{l}\text { Uncertainty Source } \\
\text { [Classification] }\end{array}$ & Description \\
\hline Flight Technical $[\mathrm{AI}]$ & $\begin{array}{l}\text { Errors produced by the onboard } \\
\text { command and control system }\end{array}$ \\
\hline Initial conditions $[\mathrm{IC}]$ & $\begin{array}{l}\text { Initial values of the aircraft state } \\
\text { variables }\end{array}$ \\
\hline Aircraft Motion Modelling & \\
\hline $\begin{array}{l}\text { Modelling intrinsic } \\
\text { [AMM] }\end{array}$ & $\begin{array}{l}\text { Errors introduced by the } \\
\text { assumptions considered in the } \\
\text { formulation of the AMM }\end{array}$ \\
\hline Solvability [AMM] & $\begin{array}{l}\text { Definition of the constraints } \\
\text { selected for obtaining solvable } \\
\text { DAE systems }\end{array}$ \\
\hline $\begin{array}{l}\text { Aircraft Performance Modelling } \\
{[\mathrm{APM}]}\end{array}$ & $\begin{array}{l}\text { Differences between actual and } \\
\text { modelled aircraft performance }\end{array}$ \\
\hline Earth model & \\
\hline $\begin{array}{l}\text { Earth surface model } \\
\text { [EWM] }\end{array}$ & $\begin{array}{l}\text { Differences between actual and } \\
\text { modelled Earth surface }\end{array}$ \\
\hline $\begin{array}{l}\text { Gravitational model } \\
\text { [EWM] }\end{array}$ & $\begin{array}{l}\text { Differences between actual and } \\
\text { modelled gravitational force }\end{array}$ \\
\hline Weather Forecast & \\
\hline Atmosphere model [EWM] & $\begin{array}{l}\text { Differences between actual and } \\
\text { forecasted atmosphere conditions }\end{array}$ \\
\hline $\begin{array}{l}\text { Altitude description } \\
\text { [AI] }\end{array}$ & $\begin{array}{l}\text { Influence of the atmosphere } \\
\text { conditions on the definition of the } \\
\text { pressure altitude }\end{array}$ \\
\hline $\begin{array}{l}\text { Airspeed description } \\
{[\mathrm{AI}]}\end{array}$ & $\begin{array}{l}\text { Influence of the atmosphere } \\
\text { conditions on the definition of the } \\
\text { airspeed }\end{array}$ \\
\hline $\begin{array}{l}\text { Wind model uncertainties } \\
\text { [EWM] }\end{array}$ & $\begin{array}{l}\text { Differences between actual and } \\
\text { forecasted wind field }\end{array}$ \\
\hline Aircraft Intent Description & \\
\hline Continuity conditions $[\mathrm{AI}]$ & $\begin{array}{l}\text { Description of the initial aircraft } \\
\text { state at any of the consecutive AI } \\
\text { operations in the sequence }\end{array}$ \\
\hline Instruction effect [AI] & $\begin{array}{l}\text { Mathematical definition of the } \\
\text { applicable constraints at each } \\
\text { individual AI operation, which lead } \\
\text { to solvable DAE systems }\end{array}$ \\
\hline Trigger [AI] & $\begin{array}{l}\text { Difference between the actual and } \\
\text { modelled trigger that defines the } \\
\text { end of an AI operation and the } \\
\text { beginning of the next one in the } \\
\text { sequence }\end{array}$ \\
\hline
\end{tabular}

For the remaining of this paper the following sources will not be modelled and, thus, will be discarded for the research presented hereafter:

- $\quad$ Flight Technical Uncertainties. These are discrepancies with respect to the predicted trajectory due to errors in the flight control system, i.e., they represent the lack of adherence to the aircraft track. It can be assumed that the long-term effect on a predicted trajectory is negligible compared with the influence of other sources of uncertainties. If this effect becomes noticeable, then the aircraft is not being operated according to its nominal capabilities and, therefore, the assumptions considered in the definition of the AMM are not valid, leading to predictions incoherent with the actual aircraft capabilities.

- $\quad$ Earth surface model. Regardless if the Earth surface is modelled with simpler (e.g., flat or round), or more sophisticated (e.g., ellipsoidal WGS84) models, the deviations with the actual Earth surface does not show a stochastic behavior and, thus, its influence on prediction uncertainty can be discarded.

- Gravitational model. Common gravitational models used by most TPs provide enough accuracy so that the error introduced is smaller in comparison with those produced by other models. In addition, its nature is nonstochastic as in the case of the Earth surface model. Hence, this bias introduced in the prediction process can be not considered.

The proposed uncertainty analysis relies on the existence of a valid AI instance representing the intended trajectory (there is a univocal relationship between an AI instance and a predicted trajectory). The process of generating the AI instance itself is affected by other different sources of uncertainty (e.g., user preferences) rather than those shown in Table IV. It has been assumed that the identified uncertainty sources affecting the AI capture such AI generation uncertainty.

\section{UQ based on a Non-intrusive PC approach}

Once the trajectory prediction model under study is determined, and the sources of uncertainty are identified, it is possible to assess how uncertainty propagates into the outputs by applying the PC theory.

The process aims at obtaining the mode strengths $b_{i}$ of the multivariate expansions that represent the prediction outcomes. There are basically two approaches [21] that can be followed:

- Intrusive approach, which proposes to substitute the inputs to the model by the related univariate PCE, and solve the system of equations to obtain the multivariate PCE representing the outputs. Galerkin projection takes advantage of the orthogonality of the polynomial basis $\psi_{i}$ to define a system of equations that returns $b_{i}$. The main drawbacks of this solution are: (a) an explicit mathematical representation of the model is a must; (b) the system of equations to be solved is of higher complexity than the original one; (c) a modification of the original solver is required to obtain the solution; (d) 
it is usually tailored to a specific model and its implementation cannot be extended to other models; and (e) if additional germs are to be considered, a reformulation of the system of equations is required.

- Non-intrusive approach, which treats the model as a black-box. Inputs are sampled to obtain the set of corresponding outputs from which the mode strengths $b_{i}$ can be obtained by regression methods. Main advantages of this solution are: (a) an explicit representation of the model is not a must; (b) mode strengths are easily obtained; (c) any modification of the multivariate PCE can be straightforward assessed; (d) it does not imply any modification of the original definition of the model specification; and (e) it can be applicable to different models by just computing the corresponding outputs of the selected sampling of inputs.

Based on the drawbacks of the intrusive approaches and the advantages of non-intrusive ones, the research presented in this paper follows the so-called non-intrusive Probabilistic Collocation Method (PCM) [22]. The PCM establishes at which collocation points $\left\{\left(\xi_{1,1}, \ldots, \xi_{N, 1}\right), \ldots,\left(\xi_{1, q}, \ldots, \xi_{N, q}\right)\right\}$ the model needs to be evaluated to obtain the intentioned set of outputs that enable the computation of the mode strengths of the PCE. The collocation points related to the germ $\xi_{i}$ are obtained as the roots of the polynomial $\psi_{d+1}\left(\xi_{i}\right)$ of next higher order than the order of the polynomial $\psi_{d}\left(\xi_{i}\right)$ at which the PCE is to be truncated. In the case of multivariate problems with $N$ independent variables, the number of collocation points reaches up to $q=(d+1)^{N}$ corresponding to the $N$ combinations of the $(d+1)$ roots of the univariate polynomial expansions.

Evaluating the model at those computed collocation points, the following system of linear equations returns the mode strengths by applying regression techniques (e.g., least square fitting).

$$
\begin{gathered}
z_{1}=\sum_{i=1}^{\infty} b_{i} \phi_{i}\left(\xi_{1,1}, \xi_{2,1}, \ldots, \xi_{N, 1}\right) \\
\vdots \\
z_{q}=\sum_{i=1}^{\infty} b_{i} \phi_{i}\left(\xi_{1, q}, \xi_{2, q}, \ldots, \xi_{N, q}\right)
\end{gathered}
$$

The number of mode strengths to be computed is $p$ as defined by (7). Thus, $q$ can be reduced up to $p$ collocation points. Any subset of $p$ elements selected from the set of $q$ leads to the definition of a solvable linear system of equations. However, different subsets will return different estimates of the mode strengths $b_{i}$. This can be overcome by selecting the $p$ from the most probable areas of the probability distributions that describe the germs $\left\{\xi_{1}, \ldots, \xi_{N}\right\}$.

The following section exemplifies how this methodology can be applied to a trajectory prediction problem through a case study.

\section{CASE STUdy}

\section{A. Description of trajectory prediction inputs}

1) Aircraft performance model. The selected aircraft type will be a Boeing 737-800 equipped with CFM56-7B26/27 engines developed by CFMI, joint-owned company of Safran Aircraft Engines and GE Aviation. The B738W26 dataset included in the release 4.1 of BADA (Base of Aircraft Data) [23] provides the required performance models for this aircraft type.

2) Weather model. The selected day of operation is 2016 February 14. Weather forecasts for such date downloaded from the National Oceanic and Atmospheric Administration (NOAA) website will be used as a representation of the weather conditions affecting the trajectory.

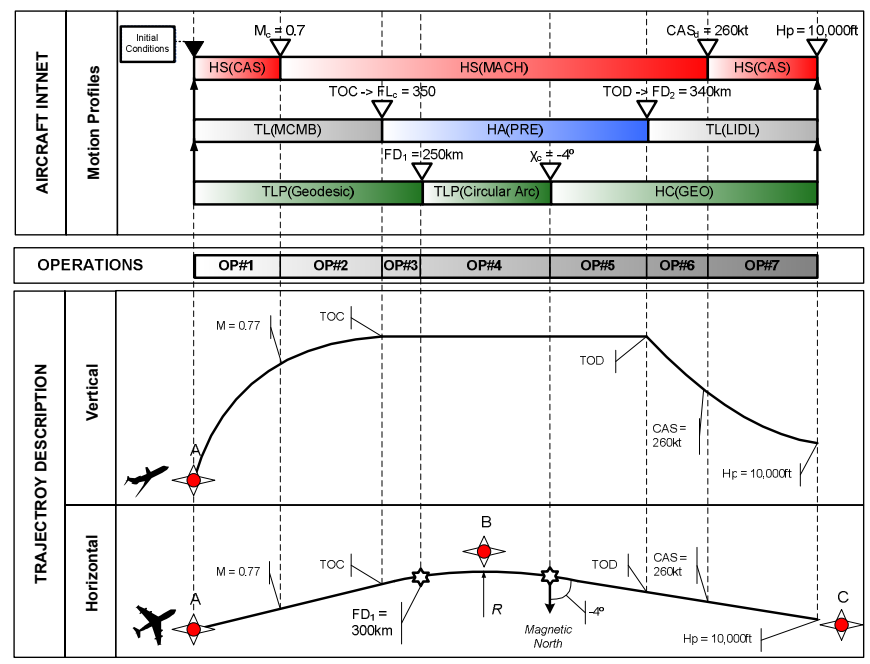

Fig. 2. AI instance \& Trajectory Description

3) Aircraft Intent description. Assuming that the trajectory to be predicted will be flown in clean configuration, it is only required to specify the motion constraints (i.e., the AIDL instructions along the lateral [horizontal plane] and the two longitudinal threads [vertical plane]). The lateral path is described by a geodesic between waypoints A and B that will be followed for $250 \mathrm{~km}$, a circular arc of radius $(\mathrm{R}=10 \mathrm{~km})$ around $\mathrm{B}$ up to capturing a bearing $X_{c}$ of $-4^{\circ}$ respect to the magnetic North, and finally a geodesic defined by the end point of the circular arc and the waypoint $\mathrm{C}$. The vertical profile is described by an initial climb at maximum climb (MCMB) engine rating and constant $\mathrm{CAS}_{0}$ up to the transition altitude at which the Mach cruise speed $\left(\mathrm{M}_{\mathrm{c}}=0.7\right)$ is reached. From this point the climb is performed at constant Mach speed until reaching the Top of Climb (TOC) at flight level FL350. This condition defines the beginning of the cruise phase, which is executed at a constant Mach number and FL. This phase ends at the Top of Descent (TOD), defined by a point $340 \mathrm{~km}$ away from the beginning of the trajectory. Following the TOD, the 
aircraft initiates the descending phase at a constant Mach speed and low idle (LIDL) engine regime up to the transition altitude at which the descent is performed at constant CAS $\left(\mathrm{CAS}_{\mathrm{d}}=\right.$ $260 \mathrm{kn})$. The trajectory ends once the aircraft reaches $10,000 \mathrm{ft}$ of pressure altitude. Fig. 2 only shows the three motion profiles required to describe the considered trajectory out of the six required as by the AIDL grammar rules. The three remaining configuration profiles will not vary due to the trajectory will be predicted at clean configuration (i.e., landing gear and flaps retracted and no activation of speed breaks at any time). The depicted triggers define events on the trajectory (e.g., TOC @ FL360) that determine the sequence of OPs (i.e., DAE system as exposed above) representing the trajectory.

4) Initial conditions. Considering the selected aircraft type and the AI description, the initial conditions that describe the first aircraft state, from which the trajectory is to be predicted, are included in following Table V.

TABLE V. INITIAL CONDITIONS

\begin{tabular}{|c|c|c|c|c|c|}
\hline $\mathbf{t}_{\mathbf{0}}$ & $\boldsymbol{\lambda}_{\mathbf{0}}$ & $\boldsymbol{\varphi}_{\mathbf{0}}$ & $\mathbf{m}_{\mathbf{0}}$ & $\mathbf{C A S}_{\mathbf{0}}$ & $\mathbf{H p}_{\mathbf{0}}$ \\
\hline $\begin{array}{c}5: 20 \\
\mathrm{GMT}\end{array}$ & $9^{\mathbf{o}} \mathrm{N}$ & $58^{\mathrm{o}} \mathrm{E}$ & 70 ton & $245 \mathrm{kt}$ & $3,000 \mathrm{ft}$ \\
\hline
\end{tabular}

\section{B. Uncertainty sources characterization.}

The characterization of potential sources of uncertainty is itself quite complex. This process can provide more realistic representations by exploiting recorded flight data or surveillance data. However, since the focus of this research is on the mathematical formulation of the trajectory prediction UQ problem, the probabilistic density functions (PDFs) proposed hereafter have been considered as sufficiently representative of the inputs variability, although, they are not built upon real data.

From all possible sources of uncertainty listed in Table IV, the following parameters have been selected, meaning that all remaining ones are not considered as contributors to the prediction uncertainty:

- Initial conditions: initial time $\left(\mathrm{t}_{0}\right)$ and initial aircraft mass $\left(\mathrm{m}_{0}\right)$

- AI Description: cruise Mach speed $\left(\mathrm{M}_{\mathrm{c}}\right)$, cruise Flight Level $\left(\mathrm{FL}_{\mathrm{c}}\right)$, capturing heading $\left(\mathrm{X}_{\mathrm{c}}\right)$, TOD location $\left(\mathrm{FD}_{2}\right)$ and descending CAS $\left(\mathrm{CAS}_{\mathrm{d}}\right)$.

- Weather: forecast uncertainty parameter $\left(F_{\text {up }}\right)$. In addition to the numerical weather predictions, NOAA includes, as a product, the associated standard deviations of the atmospheric variables at each grid point. They are computed by intentionally perturbing the initial atmosphere state and the mathematical models to assess the impact of stochastic effects on the forecast. Considering that initially all possible weather forecasts are a priori equi-probable, the variability of the atmospheric conditions can be represented by uniform distributions. Normalizing all distributions to a uniform distribution in the interval $[-1,1]$, it is possible to model the weather uncertainty by a unique parameter $F_{\text {up }}$ defined within such interval.

- APM: independent coefficient of the drag polar model $\left(\mathrm{d}_{0}\right)$ and independent coefficient of the non-idle fuel consumption model $\left(\mathrm{f}_{0 \mathrm{i}}\right)$ as defined by the BADA 4.1 specification.

To exemplify the proposed UQ process, Table VI shows the three PDF types used to characterize the variability of the selected sources. Uniform distributions are used to characterize sources whose values are equi-probable within a range. Triangular distributions are used to represent also a variability within an interval, although unlike uniform distributions, in this case the mode (most likely value) is known. Finally, Gaussian distributions are used for those parameters assumed as normally distributed.

TABLE VI. PROBABILITY DistRIBUTION THAT CHARACTERIZE THE UNCERTAINTY SOURCES

\begin{tabular}{|c|c|}
\hline Source of Uncertainty & $\begin{array}{c}\text { Probability Density } \\
\text { Function }\end{array}$ \\
\hline$t_{0}[\mathrm{sec}]$ & $\mathcal{N}(30,5)^{\mathrm{a}}$ \\
\hline$m_{0}[\mathrm{ton}]$ & $\mathrm{UNI}(71.5,68.5)^{\mathrm{b}}$ \\
\hline$M_{c}[-]$ & $\mathrm{UNI}(0.71,0.68)$ \\
\hline$F L_{c}[\mathrm{FL}]$ & $\mathcal{N}(350,5)$ \\
\hline$\chi_{c}[\mathrm{deg}]$ & $\mathcal{N}(-4,0.33)$ \\
\hline$d_{2}[\mathrm{~km}]$ & $\mathcal{N}(340,5)$ \\
\hline$C A S_{d}[\mathrm{kn}]$ & $\mathcal{N}(260,3)$ \\
\hline$F_{u p}[-]$ & $\mathrm{UNI}(-1,+1)$ \\
\hline$d_{0} \cdot 10^{5}[-]$ & TRI $(-5.5335,-5.5331)^{\mathrm{c}}$ \\
\hline$f_{0 i}[-]$ & $\mathrm{TRI}(0.03,0.0628)$ \\
\hline${ }^{\mathrm{a}} \cdot \mathcal{N}(\mu, \sigma)-$ Gaussian distribution of mean $\mu$ and standard deviation $\sigma$ \\
\hline c. TRI(a,b) - Triangular distribution between $a$ and $b$ with mode $a$
\end{tabular}

\section{Monte Carlo benchmark}

Monte Carlo processes are a broad class of uncertainty propagation algorithms that rely on repeated random samplings to obtain numerical results. They are very easy to implement, although they are highly computationally demanding and time consuming. The accuracy of these methods respect to the actual system response improves with the inverse of the square root of the number of runs $n(1 / \sqrt{n})$. Hence, to reduce the error between the simulated and actual system outcomes by a half, it is required to increase the number of runs by four. The Stochastic Trajectory Predictor (S-TP) [24] proposed by Navarro and Valls is an example of this type of approach.

However, this approach is very useful to obtain an UQ benchmark to compare the outputs from the PC approach with. The computed mean values and associated standard deviations at the end of each AI operation are included in Table VII for the following list of state variables: elapsed time $(\mathrm{t})$, flown distance (FD), aircraft mass (m), pressure altitude (Hp), ground speed 
(Vg), calibrated airspeed (CAS) and Mach Number (M). They have been obtained from a 3,000-run Monte Carlo simulation.

TABLE VII. MONTE CARLO BENCHMARK

\begin{tabular}{|c|c|c|c|c|c|c|c|c|}
\hline \multicolumn{2}{|c|}{ OP\# } & $\begin{array}{c}\text { t } \\
{[\mathbf{s e c}]}\end{array}$ & $\begin{array}{c}\text { FD } \\
{[\mathbf{k m}]}\end{array}$ & $\begin{array}{c}\text { m } \\
{[\text { ton] }}\end{array}$ & $\begin{array}{c}\text { Hp } \\
{[\mathbf{F L}]}\end{array}$ & $\begin{array}{c}\text { Vg } \\
{[\mathbf{k n}]}\end{array}$ & $\begin{array}{c}\text { CAS } \\
{[\mathbf{k n}]}\end{array}$ & $\begin{array}{c}\text { M } \\
{[-]}\end{array}$ \\
\hline \multirow{2}{*}{$\boldsymbol{1}$} & $\boldsymbol{\mu}$ & 665 & 110 & 68.87 & 328 & 383 & 245 & 0.695 \\
\cline { 2 - 9 } & $\boldsymbol{\sigma}$ & 27 & 5.4 & 0.84 & 6.0 & 5.7 & 0.0 & $9 \mathrm{e}-3$ \\
\hline \multirow{2}{*}{$\boldsymbol{2}$} & $\boldsymbol{\mu}$ & 739 & 124 & 68.79 & 350 & 384 & 233 & 0.695 \\
\cline { 2 - 9 } & $\boldsymbol{\sigma}$ & 25 & 5.0 & 0.84 & 4.8 & 5.5 & 4.0 & $9 \mathrm{e}-3$ \\
\hline \multirow{2}{*}{3} & $\boldsymbol{\mu}$ & 1,379 & 250 & 68.42 & 350 & 383 & 233 & 0.695 \\
\cline { 2 - 9 } & $\boldsymbol{\sigma}$ & 15 & 0.0 & 0.84 & 4.8 & 5.5 & 4.0 & $9 \mathrm{e}-3$ \\
\hline \multirow{2}{*}{4} & $\boldsymbol{\mu}$ & 1,555 & 285 & 68.31 & 350 & 413 & 233 & 0.695 \\
\cline { 2 - 9 } & $\boldsymbol{\sigma}$ & 17 & 0.1 & 0.84 & 4.8 & 5.5 & 4.0 & $9 \mathrm{e}-3$ \\
\hline \multirow{2}{*}{5} & $\boldsymbol{\mu}$ & 1,819 & 340 & 68.16 & 350 & 408 & 233 & 0.695 \\
\cline { 2 - 9 } & $\boldsymbol{\sigma}$ & 30 & 5.0 & 0.84 & 4.8 & 5.4 & 4.0 & $9 \mathrm{e}-3$ \\
\hline \multirow{2}{*}{$\boldsymbol{6}$} & $\boldsymbol{\mu}$ & 2,024 & 383 & 68.14 & 301 & 408 & 260 & 0.695 \\
\cline { 2 - 9 } & $\boldsymbol{\sigma}$ & 58 & 9.4 & 0.84 & 8.2 & 5.8 & 3.0 & $9 \mathrm{e}-3$ \\
\hline \multirow{2}{*}{7} & $\boldsymbol{\mu}$ & 3,273 & 598 & 67.93 & 100 & 283 & 260 & 0.470 \\
\cline { 2 - 9 } & $\boldsymbol{\sigma}$ & 38 & 7.1 & 0.84 & 0.0 & 4.2 & 3.0 & $5 \mathrm{e}-3$ \\
\hline
\end{tabular}

\section{D. aPC approach: numerical results}

The aPCE method, as exposed in Section III-B, only requires the computation of the statistical moments up to order $2 k-1$ (being $k$ the order of the polynomial expansion) of the PDFs shown in Table VI representing each individual source of uncertainty.

According to (7), the number of mode strengths $(p)$ will depend on the selected order of the PCE $(d)$ and the number of uncertainty sources $\xi_{i}$ (i.e., germs). In the proposed case study, the number of considered inputs $N$ is 11 , while $d$ needs to be selected.

To assess the accuracy of the proposed methodology with respect to the benchmark, polynomial expansions of order $k=$ $\{1,2,3\}$ have been evaluated.

The relative RMS errors (i.e., difference between Monte Carlo and aPCE results referred to the Monte Carlo outputs) have been calculated defined by (19) for three selected expansion orders, where $\chi$ represents the mean and standard deviation of the studied state variables at the 7 OPs that identify the AI segments between two consecutive trajectory events.

$$
\chi_{R M S E}=\sqrt{\frac{\sum_{r=1}^{7}\left(\frac{\chi_{M C}^{r}-\chi_{a P C E}^{r}}{\chi_{M C}^{r}}\right)^{2}}{7}} \cdot 100
$$

Following Table VIII show the numerical results for the three expansions under consideration.
TABLE VIII. RELATIVE RMS ERRORS

\begin{tabular}{|c|c|c|c|c|c|c|c|}
\hline & $\begin{array}{c}\mathbf{t}_{\text {RMSE }} \\
{[\%]}\end{array}$ & $\begin{array}{c}\text { FD } \\
\text { RMSE } \\
{[\%]}\end{array}$ & $\begin{array}{c}\text { m RMSE } \\
{[\%]}\end{array}$ & $\begin{array}{c}\text { Hp } p_{\text {RMSE }} \\
{[\%]}\end{array}$ & $\begin{array}{c}\text { Vgrase } \\
{[\%]}\end{array}$ & $\begin{array}{c}\text { CAS }_{\text {RMSE }} \\
{[\%]}\end{array}$ & $\begin{array}{c}\text { M RMSE } \\
{[\%]}\end{array}$ \\
\hline \multicolumn{8}{|c|}{ aPCE-1 $(k=1)$} \\
\hline$\mu$ & $5 e-2$ & $6 e-2$ & $6 e-3$ & $4 \mathrm{e}-3$ & $5.6 \mathrm{e}-2$ & $1.3 \mathrm{e}-2$ & $1.3 e-3$ \\
\hline$\sigma$ & 2.7 & 2.8 & 0.13 & 0.18 & 1.2 & 1.8 & $3.9 \mathrm{e}-2$ \\
\hline \multicolumn{8}{|c|}{$a P C E-2(k=2)$} \\
\hline$\mu$ & $7 e-2$ & $7 e-2$ & $7 \mathrm{e}-3$ & $2.2 \mathrm{e}-3$ & $3 e-3$ & $3.2 \mathrm{e}-3$ & $1.3 \mathrm{e}-3$ \\
\hline$\sigma$ & 2.0 & 1.83 & 0.10 & 0.22 & 0.68 & 1.7 & $3.9 \mathrm{e}-2$ \\
\hline \multicolumn{8}{|c|}{$a P C E-3(k=3)$} \\
\hline$\mu$ & $9 \mathrm{e}-2$ & $9 \mathrm{e}-2$ & $1.8 \mathrm{e}-2$ & $2.2 \mathrm{e}-3$ & $4.5 \mathrm{e}-3$ & $3.2 \mathrm{e}-3$ & $1.3 \mathrm{e}-3$ \\
\hline$\sigma$ & 2.0 & 1.57 & $7.4 \mathrm{e}-2$ & 0.23 & 0.56 & 1.7 & $3.9 \mathrm{e}-2$ \\
\hline
\end{tabular}

\section{E. Discussion}

From the analysis of the relative RMS errors shown in previous Table VIII, it can be deducted that the mean values of the considered state variables computed using aPCE at the end of the aircraft intent operations matches perfectly those obtained from the Monte Carlo simulation. There are no meaningful differences between the 3 selected expansions, which return deviation lower than $0.1 \%$. To illustrate how accurately the expansions of different orders match the Monte Carlos outputs, the following Table IX shows the mean values of the considered state variables at the end of the trajectory prediction.

TABLE IX. COMPARISON OF MEAN VALUES AT TRAJECTORY END

\begin{tabular}{|c|c|c|c|c|c|c|c|}
\hline & $\begin{array}{c}\mathbf{t} \\
{[\mathbf{s e c}]}\end{array}$ & $\begin{array}{c}\text { FD } \\
{[\mathbf{k m}]}\end{array}$ & $\begin{array}{c}\mathbf{m} \\
{[\text { ton] }}\end{array}$ & $\begin{array}{c}\text { Hp } \\
{[\text { [FL] }}\end{array}$ & $\begin{array}{c}\text { Vg } \\
{[\mathbf{k n}]}\end{array}$ & $\begin{array}{c}\text { CAS } \\
{[\mathbf{k n}]}\end{array}$ & $\begin{array}{c}\mathbf{M} \\
{[-]}\end{array}$ \\
\hline $\boldsymbol{M C}$ & 3,273 & 597.7 & 67.93 & 100 & 282.8 & 259.9 & 0.470 \\
\hline $\boldsymbol{a P C E}-1$ & 3,271 & 597.5 & 67.94 & 100 & 282.7 & 259.9 & 0.470 \\
\hline $\boldsymbol{a P C E}-2$ & 3,270 & 597 & 67.90 & 100 & 282.9 & 259.9 & 0.470 \\
\hline $\boldsymbol{a P C E}-3$ & 3,278 & 598.5 & 67.90 & 100 & 282.8 & 259.9 & 0.470 \\
\hline
\end{tabular}

The standard deviations computed from the aPCE, which can be considered as a measurement of the prediction uncertainty, show also a very high accuracy for all the expansions (lower than 3\%). Next Table $\mathrm{X}$ includes the values of the computed standard deviations at the end of the trajectory prediction for the studied state variables.

TABLE X. COMPARISON OF STANDARD DEVIATIONS AT TRAJECTORY END

\begin{tabular}{|c|c|c|c|c|c|c|c|}
\hline & $\begin{array}{c}\mathbf{t} \\
{[\mathbf{s e c}]}\end{array}$ & $\begin{array}{c}\text { FD } \\
{[\mathbf{k m}]}\end{array}$ & $\begin{array}{c}\mathbf{m} \\
{[\mathbf{t o n}]}\end{array}$ & $\begin{array}{c}\text { Hp } \\
{[\mathbf{F L}]}\end{array}$ & $\begin{array}{c}\text { Vg } \\
{[\mathbf{k n}]}\end{array}$ & $\begin{array}{c}\text { CAS } \\
{[\mathbf{k n}]}\end{array}$ & $\begin{array}{c}\text { M } \\
{[-]}\end{array}$ \\
\hline $\boldsymbol{M C}$ & 37.8 & 7.1 & 0.839 & 0 & 4.16 & 3.04 & $5 \mathrm{e}-3$ \\
\hline $\boldsymbol{a P C E}-1$ & 38.7 & 7.14 & 0.841 & 0 & 4.19 & 3.04 & $5 \mathrm{e}-3$ \\
\hline $\boldsymbol{a P C E}-2$ & 39.3 & 7.32 & 0.837 & 0 & 4.11 & 3.04 & $5 \mathrm{e}-3$ \\
\hline $\boldsymbol{a P C E}-3$ & 39.5 & 7.2 & 0.855 & 0 & 4.12 & 3.04 & $5 \mathrm{e}-3$ \\
\hline
\end{tabular}

In addition to the accuracy of the expansions, the main advantage of the aPCE approach is the low computational 
requirements in terms of number of trajectory predictions $\left(n_{t p}\right)$ and execution interval $\left(\mathrm{e}_{i}\right)$ compared to traditional Monte Carlo simulations. Following Table IX compares the number of Monte Carlo predictions and the associated computational effort with those values obtained from the selected polynomial chaos expansions.

TABLE XI. COMPARISON OF COMPUTATIONAL REQUIREMENTS

\begin{tabular}{|c|c|c|c|c|}
\hline & MC & aPCE-1 & aPCE-2 & aPCE-3 \\
\hline $\boldsymbol{n}_{\boldsymbol{t} p}$ & 3,000 & 11 & 66 & 286 \\
\hline $\mathbf{e}_{\boldsymbol{i}}[\mathbf{s e c}]$ & 399 & 1.8 & 8.5 & 42.4 \\
\hline
\end{tabular}

The efficiency of the proposed aPCE approach grows as the order of the expansions decreases. However, regardless the selected order, the benefits and time savings with respect to the Monte Carlo simulations are obvious in all cases.

According the exposed results, it can be concluded that the aPCE approach provides very accurate quantification of trajectory prediction uncertainties (represented by the mean and the standard deviation) for any of the aircraft state variables along the whole trajectory. In this particular use case, the best alternative would be to choose expansions of order 1 due to its high accuracy and very low computational requirements. However, this could be different depending on the number of sources of uncertainty and the probabilistic density functions that characterize them [25].

Based on the definition of the proposed case study, the theoretical representation of inputs uncertainty and the accuracy results provided in Table VIII, it can be concluded that PC expansions of order 1 return extremely accurate representations of the prediction uncertainty with very low computational requirements.

\section{REMARKS AND FUTURE STEPS}

The research presented herein proposes a novel approach to quantify the uncertainty associated to any aircraft trajectory prediction. This approach is based on the application of the PC theory to the trajectory prediction process. Once the sources of uncertainty are identified and characterized, it is possible to represent their stochasticity by means of orthonormal univariate polynomial expansions. From them, the proposed approach derives the multivariate expansions that represent the uncertainty of the aircraft state variables along the prediction.

The numerical results suggest that it is possible to obtain very accurate representations of the prediction uncertainty with expansion of low order with significant computational savings compared to a 3,000-run Monte Carlo simulation (up to 200 times faster in the case of using expansions of order 1). This method provides high accuracy computing both the mean and standard deviations of all aircraft state variables throughout a complete trajectory.

The potential benefits that can be obtained from the application of an aPCE-based uncertainty quantification are manifold:
- This solution can potentially be applied to any trajectory predictor without modifying its native implementation.

- It is a fast and computationally efficient procedure, especially when compared with classical approaches like Monte Carlo. It can be considered as a pseudo-real time process taking into account typical look-ahead of the DST that rely on trajectory predictions.

- It is a data-driven process, that is, analytical representations of the probability distributions characterizing the sources of uncertainty are not required.

- It provides analytical descriptions of prediction uncertainties built upon polynomial expansions that can be easily processed by computer-based CDM processes.

Next stages of this research will aim at exploiting actual flight data or surveillance data to characterize more realistically the sources of uncertainty.

\section{ACKNOWLEDGMENTS}

The authors wish to appreciate the contribution of Dr. Sergey Oladyshkin, who strongly helped us with the development of the PC-based trajectory prediction tool used in this work.

\section{REFERENCES}

[1] Commission of the European Communities. (1996). White Paper: Air Traffic Management - Freeing Europe's Airspace. COM (96) 57 Final.

[2] Mukai, C., \& Hunter, G. (1996, July). A higher fidelity point-mass simulation of aircraft dynamics. In AIAA Guidance, Navigation and Control Conference.

[3] Walker, W. E., Harremoës, P., Rotmans, J., van der Sluijs, J. P., van Asselt, M. B., Janssen, P., \& Krayer von Krauss, M. P. (2003). Defining uncertainty: a conceptual basis for uncertainty management in modelbased decision support. Integrated assessment, 4(1), 5-17.

[4] EUROCONTROL. (2003 Edition). ATM Strategy for the Years 2000+.

[5] JPDO. (June 2007). NextGen Concept of Operations V2.0.

[6] Australian Strategic Air Traffic Management Group (ASTRA). (2007). The ATM Strategic Plan (Part A).

[7] Mondoloni, S., \& Swierstra, S. (2005, October). Commonality in disparate trajectory predictors for air traffic management applications. In Digital Avionics Systems Conference, 2005. DASC 2005. The 24th (Vol. 1, pp. 3-C). IEEE.

[8] Torres, S. (2015). Trajectory Accuracy Sensitivity to Modeling Factors. In 15th AIAA Aviation Technology, Integration, and Operations Conference (ATIO).

[9] Ryan, H. F., Paglione, M., \& Green, S. M. (2004, August). Review of trajectory accuracy methodology and comparison of error measurement metrics. In Proceedings American Institute of Aeronautics and Astronautics (AIAA) Guidance, Navigation, and Control Conference, Providence, Rhode Island.

[10] Torres, S. (2010, October). Determination and ranking of trajectory accuracy factors. In Digital Avionics Systems Conference (DASC), 2010 IEEE/AIAA 29th (pp. 1-C). IEEE.

[11] Jackson, M. R., Zhao, Y. J., \& Slattery, R. A. (1999). Sensitivity of trajectory prediction in air traffic management. Journal of Guidance Control and Dynamics, 22, 219-228.

[12] O'Hagan, A. (2013). Polynomial Chaos: A tutorial and critique from a statistician's perspective. SIAM/ASA J. Uncertainty Quantification, 20, 120. 
[13] Xiu, D., \& Karniadakis, G. E. (2002). The Wiener--Askey polynomial chaos for stochastic differential equations. SIAM journal on scientific computing, 24(2), 619-644.

[14] Xiu, D., \& Karniadakis, G. E. (2003). Modeling uncertainty in flow simulations via generalized polynomial chaos. Journal of computational physics, 187(1), 137-167.

[15] Li, H., \& Zhang, D. (2007). Probabilistic collocation method for flow in porous media: Comparisons with other stochastic methods. Water Resources Research, 43(9).

[16] Wan, X., \& Karniadakis, G. E. (2006). Multi-element generalized polynomial chaos for arbitrary probability measures. SIAM Journal on Scientific Computing, 28(3), 901-928.

[17] Oladyshkin, S., Class, H., Helmig, R., \& Nowak, W. (2011). A concept for data-driven uncertainty quantification and its application to carbon dioxide storage in geological formations. Advances in Water Resources, 34(11), 1508-1518.

[18] Oladyshkin, S., \& Nowak, W. (2012). Data-driven uncertainty quantification using the arbitrary polynomial chaos expansion. Reliability Engineering \& System Safety, 106, 179-190.

[19] Lopez-Leones, J., Vilaplana, M. A., Gallo, E., Navarro, F. A., \& Querejeta, C. (2007, October). The aircraft intent description language: A key enabler for air-ground synchronization in trajectory-based operations.
In Digital Avionics Systems Conference, 2007. DASC'07. IEEE/AIAA 26 th (pp. 1-D). IEEE.

[20] Lopez Leones, J. (2008). Definition of an aircraft intent description language for air traffic management applications (Doctoral dissertation, University of Glasgow).

[21] Onorato, G., Loeven, G. J. A., Ghorbaniasl, G., Bijl, H., \& Lacor, C. (2010, June). Comparison of intrusive and non-intrusive polynomial chaos methods for CFD applications in aeronautics. In $V$ European Conference on Computational Fluid Dynamics ECCOMAS, Lisbon, Portugal (pp. 14-17).

[22] Shi, L., Yang, J., Zhang, D., \& Li, H. (2009). Probabilistic collocation method for unconfined flow in heterogeneous media. Journal of hydrology, 365(1), 4-10.

[23] EUROCONTROL. "Base of Aircraft Data (BADA)." Eurocontrol Research \& SESAR website. 2015. Last accessed on January 29, 2017. https://www.eurocontrol.int/services/bada.

[24] Navarro, F., Valls, E. H., Vilaplana, M., Chessa, P., \& Massaveu, C. Q. (2014). U.S. Patent Application No. 15/109,319.

[25] Casado Magaña, E. J. (2016). Trajectory prediction uncertainty modelling for Air Traffic Management (Doctoral dissertation, University of Glasgow). 